\title{
Coupling Genotyping and Computational Modeling in Prediction of Anti- epileptic Drugs that cause Stevens Johnson Syndrome and Toxic Epidermal Necrolysis for Carrier of HLA-B*15:02
}

\author{
Lay Kek Teh ${ }^{1,2}$, Manikandan Selvaraj ${ }^{1}$, Zakaria Bannur ${ }^{1}$, Mohd Ikhwan Mohd Ismail ${ }^{1}$, Md. Hanip Rafia ${ }^{3}$, Wan Chung \\ $\mathrm{Law}^{3}$, Sapiah Sapuan ${ }^{3}$, Santhi Puvanarajah ${ }^{3}$, Pakeer Shaik Syed Ali ${ }^{1}$, Mohd Zaki Salleh ${ }^{1,2}$ \\ ${ }^{1}$ Integrative Pharmacogenomics Institute, Universiti Teknologi MARA Selangor, Puncak Alam, Malaysia; ${ }^{2}$ Faculty of
Pharmacy, Universiti Teknologi MARA Selangor, Puncak Alam, Malaysia; ${ }^{3}$ Neurology Institute, Hospital Kuala Lumpur,
Malaysia
}

Received, December 4, 2015; Revised, March 16, 2016; Accepted, Match 21, 2016; Published, March 21, 2016.

\begin{abstract}
Purpose. The importance of $H L A-B * 15: 02$ genotyping to avoid carbamazepine induced SJS/TEN and molecular modeling to predict the role of $H L A-B * 15: 0$ and AEDs induced SJS/TEN are investigated. Methods. DNA was extracted from eighty-six patients. The patients were genotyped by AS-PCR. Computational modeling of the $H L A-B * 15: 02$ followed by docking studies were performed to screen 26 AEDs that may induce ADR among $H L A-B^{*}$ 15:02 carriers. Results. Odd ratio for CBZ induced SJS/TEN and $H L A$ $B^{*} 15$ :02 was 609.0 (95\% CI: 23-15873; $\left.p=0.0002\right)$. Molecular modeling studies showed that acetazolamide, ethosuxiamide, lamotrigine, oxcarbazepine, phenobarbital, phenytoin, primidone and sodium-valproate may induce ADR in $H L A-B^{*}$ 15:02 carriers alike CBZ. Conclusion. We confirmed $H L A-B * 15: 02$ as a predictor of $\mathrm{SJS} / \mathrm{TEN}$ and recommend pre-screening. Computational prediction of DIHR is useful in personalized medicine.

This article is open to POST-PUBLICATION REVIEW. Registered readers (see "For Readers") may comment by clicking on ABSTRACT on the issue's contents page.
\end{abstract}

\section{INTRODUCTION}

Drug-induced hypersensitivity reactions (DIHR) such as Stevens-Johnson syndrome (SJS) and toxic epidermal necrolysis (TEN) have resulted in high mortality and lowered the quality of life of patients. The most commonly studied example is carbamazepine (CBZ) induced SJS/ TEN. The pathophysiological mechanisms underlying these two fatal cutaneous drug reactions, however, are not well understood.

Several hypotheses have been established explaining how CBZ induces SJS and TEN. Oxidative metabolites such as epoxide were thought to be the culprit of serious cutaneous reactions [1]. Many studies have suggested that CBZ might affect the antioxidant capacity of an individual but the mechanism by which CBZ affects the oxidantantioxidant balance of an individual and its association with adverse drug reaction (ADR) remained unclear [2-4]. However, an immune pathogenesis is most likely as drug-specific T cells had been identified in patients who experience SJS/TEN [5]. With reference to the hapten/prohapten hypothesis, the active chemical molecule forms a covalent bond with an endogenous peptide, which is intracellularly processed and presented by HLA [6]. The p-i concept (direct pharmacologic interaction of a drug with immune receptors) implies that the direct and reversible interaction of drugs with TCRs or peptide-loaded HLA initiates immune reactions [7]. Nevertheless, there is very few evidence that prove these hypotheses. Several theories postulate the drug-driven antigenic stimulation of immunologic receptors in drug induced hypersensitivity. Studies on HLA class I alpha $(\alpha)$ chain refolding assay showed the direct interaction of eight synthesized nanomer peptides with HLA class I $\alpha$ chains.

\footnotetext{
Corresponding Authors: Lay Kek Teh (tehlaykek2016@gmail.com) or Mohd Zaki Salleh (zakisalleh.mzs@gmail.com), Integrative Pharmacogenomics Institute (iPROMISE), Level 7, FF3, Universiti Teknologi MARA Malaysia Selangor, Puncak Alam Campus, 42300 Puncak Alam, Selangor, Malaysia.
} 
Out of the eight nanomer peptides, B27 $(\mathrm{B} * 2705)$ peptide was derived from binding heat shock protein peptide HSP89 $\alpha(201-209)$ and the other seven were derived from the human tumor suppressor protein p53 sequence. Testing a total of 36 allospecific HLA class-I protein have shown that the majority of these peptides possess ArginineLysine (ARG-LYS) motif that firmly binds to $\alpha$ chains of B27 but binds weakly to A31 $\alpha$ chains. Whereas, the peptides of p53 (173-181) VRRCPHHER and (334-342) GRERFEMFR with the ARG-ARG motif showed a considerable binding affinity to A31 $\alpha$ chains but not with other $\alpha$ chains. This depicts both the specificity and the cross-peptide-binding of peptides to HLA allelic products of different HLA loci [8]. A previous study has shown that CBZ binds to $H L A-B * 15: 02$ that results in an alteration in the repertoire of the presented self-peptides [9].

Involvement of the major histocompatibility complex for CBZ induced cutaneous reaction has been known ; 100\% of Han Chinese patients with carbamazepine-induced SJS/TEN were positive for human leukocyte antigen $H L A-B * 15: 02$ allele, compared with $8 \%$ in the healthy population [10, 11]. These DIHRs are significantly correlated with $H L A-B * 15: 02$ polymorphism which is common in Thailand, India, Malaysia, Hong Kong, and in descendants of immigrants from Southeast Asia [12]. A meta-analysis performed by Tangamornsuksan et al. reported that in Thailand, Singapore and Malaysia, usage of carbamazepine represents up to one-third of all SJS and TEN cases [13].The firm association between human leukocyte antigen $H L A-B^{*} 15: 02$ and carbamazepine-induced SJS in Asians is however not seen among the Europeans [14]. Similarly, some studies among the Japanese reported lack of association with HLA$B^{*}$ 15:02 [15,16]. Evidence from the published literature supporting this relationship was summarised, and recommendations for the use of carbamazepine based on $H L A-B$ genotype was provided by The PharmGKB (http://www.pharmgkb.org) which is a publicly available Web-based knowledge base [17]. The guideline suggested that carbamazepine should not be recommended for carbamazepine-naive individuals who have at least one copy of the HLA$B * 15: 02$ allele. If a patient has previously used carbamazepine for longer than three months without incidence of cutaneous adverse reactions, consider cautious use of carbamazepine if no alternative is available. Besides, , cautious use of alternative AEDs is recommended, but there is a lack of evidence linking serious cutaneous ADR induced by alternative AEDs with $H L A-B * 15: 02$ allele.

In this study, the association of $H L A-B^{*} 15: 02$ and CBZ induced SJS/TEN is investigated using the in-house developed PCR-based detection method. Two different perspective groups of patients were genotyped; a retrospective group with patients prescribed CBZ and developed SJS/TEN and another prospective group in which patients were genotyped before CBZ therapy was started. The retrospective group allows the odd ratios to be calculated while the prospective group provide insights to the importance of pre-genotyping among patients. In addition, we have developed three dimensional structural models for $H L A-B * 15: 02$ using homology modeling approach and endogenous peptide interaction to the binding site of the above model was done through peptide docking studies. Subsequently, docking studies were used to screen the AEDs that may cause drug induced hypersensitivity similar to CBZ, which are associated with $H L A-B^{*} 15: 02$ allele. Our study implies the hapten/prohapten hypothesis, the chemical active molecule forms a covalent bond with an endogenous peptide, which is intracellularly processed and presented by HLA to induce ADR. The data generated will add more confidence to the local practitioners on the impact of pharmacogenomics based clinical practises.

\section{METHODS}

\section{Subjects and sample collection}

Approval from the constitutional Human Research Ethics Committee and Medical Research and Ethics Committee (MREC) was obtained. Eighty six (86) patients attending clinics at the Department of Neurology, in a local hospital were recruited and written informed consents were obtained from all patients. This is a single masked (Single-blind) study where the patients were randomly selected by the medical officers attending patients at the Department of Neurology, based on the inclusion and exclusion criteria. The technician who performed the pharmacogenetic test did not know the clinical status of the patients involved in this study. Patients were recruited if they were 18-55 years old, mentally healthy, on CBZ treatment for bipolar, neurologic pain or epilepsy at the department of Neurology, understand the study 
protocol and able to provide informed consent. Patients who did not understand the study protocol, unable to follow simple instructions or were not willing to provide signed informed consent were excluded. Subjects in the study were informed of the details of the study before giving their consent. Blood $(5 \mathrm{ml})$ was taken from the patient by authorized trained personnel and DNA was extracted from the blood using the salting out method as described earlier[10]. Relevant clinical data was collected by the medical officers and revealed after the test was obtained.

The patients were categorized into prospective and retrospective groups. Among the 86 patients, 40 were newly registered patients and were genotyped before the patients being prescribed CBZ (prospective).The other 46 patients who were already receiving $\mathrm{CBZ}$ at the time of entry into the study were randomly selected by the doctor for $H L A B^{*}$ 15:02 screening and they comprised the retrospective study group. The event of SJS/TEN was analyzed in relation to $H L A-B^{*} 15: 02$ genotype.

\section{HLA genotyping}

$H L A-B * 15: 02$ genotype was determined by polymerase chain reaction (PCR) using sequence specific primers. The PCR based detection method of $H L A-B * 15: 02$ allele was adopted and modified from Man et al [12]. For each PCR run, both positive and negative controls were included as quality assurance as well as to avoid misinterpretation of the result. The positive control (DNA fragment containing the four nucleotide change denoting $H L A-B^{*} 15: 02$ was inserted into a plasmid. DNA samples were then randomly selected and sent to a third party for direct sequencing to verify the accuracy of the method.

\section{STATISTICAL ANALYSIS}

The frequencies of $H L A-B * 15: 02$ was calculated. Statistical analysis (Fisher's Exact Test and Chisquare test) was performed using SPSS software version 20. $P$ values $\leq 0.05$ were considered statistically significant. The strength of association between $H L A-B * 15: 02$ with CBZ-induced SJSTEN was estimated by calculating the odds ratio.

\section{Computational studies}

\section{Homology modeling of HLA-B*15:02 structure}

The protein sequence for HLA-B*15:02 (accession no: C5J3Z8) was retrieved from the universal protein resource (UniProt), consisting of 362 amino acids [18]. The template for homology modeling was identified by PDB-BLAST (http://www.ncbi.nlm.nih.gov/blast/) available at the National Center for Biotechnology Information (NCBI). The HLA-B*15:01 structure (PDB ID: 1XR8 at $2.30 \AA$ resolution) [19] with a sequence identity of $98.19 \%$ to HLA-B*15:02 was identified as a template. Based on the template identified, the homology structure for HLA-B*15:02 was modeled using MODELLER9v11[20]. MODELLER can simultaneously incorporate structural information from one or more reference proteins. Structural topologies in the reference proteins were used to derive spatial restraints, which are used to build model protein structures by using conjugate gradient and simulated annealing optimization procedures. Among the models generated by MODELLER, the best model was selected based on the lowest generated discrete optimized protein energy (DOPE) score.

Further, the quality and stereochemistry of the models were evaluated using the program PROCHECK, where the final model was selected based on the stereochemical quality [21] and RAMPAGE Ramachandran plot

(http://mordred.bioc.cam.ac.uk/ rapper/rampage.ph p). The main-chain conformations for all amino acid residues were checked for the favored or allowed regions of the Ramachandran plot.

\section{Modeling of the peptide-loaded HLA-B*15:02 complex}

In order to model the peptide pp5 (FLFDGSPTY) loaded antigen binding groove (B-pocket) of HLA$\mathrm{B}^{*}$ 15:02, the peptide of human UbcH6 and Epstein - Barr virus EBNA-3 in complex with HLAB*15:01 (PDB ID: 1XR8) was used as a reference [22]. Further, the modeled FLFDGSPTY peptide conformation in agreement with the co-crystallized peptide of HLA-B*15:01 was visually inspected. The FLFDGSPTY peptide loaded HLA-B*15:02 complex was further optimized using FlexPepDock, a high-resolution peptide docking protocol server $[23,24]$. The complex was optimized using Rosetta FlexPepDock as mentioned above and the best energy threshold complex model was selected in each case for further studies.

PatchDock tool [25] (utilizes PDB coordinate files as input with default parameters) was used to generate HLA-peptide loaded complex mentioned above, subsequently, the FireDock program was 
used to perform energy minimization of the best models to get stable HLA-peptide complex [26] as a validating process. Each candidate transformation was evaluated by scoring function considering both geometric fit and atomic desolvation energy [27]. The candidate solutions were clustered using Root Mean Square Deviation (RMSD), discarding the redundant solutions.

\section{Docking studies on the peptide loaded complexes} The 3D structures of 26 AEDs reported by the Epilepsy Society of UK [28] were obtained from the NCBI PubChem database and their structures were optimized using Openeye software (OpenEye Scientific Software Inc., Santa Fe, NM, USA). Further, the AEDs were docked to peptide loaded complex of HLA-B*15:02 as a pre-screening process to select the possible AED candidates that may induce hypersensitivity similar to CBZ. The entire docking studies were performed using Gold (Genetic Optimization for Ligand Docking, version 5.1) [29], considering the antigen binding site (Bpocket) as the target site with a cut off of $12 \AA$ radius with $100 \%$ genetic algorithm (GA), using ChemScore as the fitness scoring function. Results divergent by less than $0.75 \AA$ in ligand-all atom root mean square deviations (RMSDs) were clustered together. Further, the binding mode of each AED candidate and their interaction patterns were analyzed by visual inspection using PyMOL [30]. During the analysis, it was prioritized that the binding mode of each AED candidate was compared with the binding mode of $\mathrm{CBZ}$ as a reference.

\section{RESULTS}

The clinical and demographic $H L A-B * 15: 02$ genotypes of the 86 patients (prospective and retrospective group) are shown in Table 1. HLA$B^{*} 15: 02$ was observed in $24.4 \%$ of the patients. No significant difference was found in terms of sex, race and indication of $\mathrm{CBZ}$ when compared with patients who carry $H L A-B * 15: 02$ ( $p$-values $>0.05$ ).

Among the patients in the prospective group, $17.5 \%$ were positive for $H L A-B^{*} 15: 02$. No significant differences were found in term of sex, race and indication of $\mathrm{CBZ}$ where the p-values from these groups are 0.273, 0.229, and 0.705 respectively.

In the retrospective group, 15 patients were of $H L A-B * 15: 02 \quad(32.6 \%)$ genotype. CBZ was withdrawn from 14of those patients due to SJS/TEN or rash and all were positive for $H L A$ $B^{*} 15: 02$. One patient with positive $H L A-B * 15: 02$, however, did not develop SJS/TEN. No significant differences in the percentage frequency of $H L A$ $B * 15: 02$ were found in the different ethnic groups or for different indications for CBZ (Table 1). However, nine patients who were detected as negative for $H L A-B * 15: 02$ developed mild rashes.

The occurrence of cADR among the patients in the retrospective group with respect to the genotype of $H L A-B^{*}$ 15:02 is shown in Table 2. The strength of association, expressed as the odds ratio for the ADR in subjects of $H L A-B * 15: 02$ genotype relative to those who were not was 609.0 (95\% CI: $23-$ 15873). $p=0.0002$.

\section{Homology modeling}

The homology model for HLA-B*15:02 was constructed using HLA-B*15:01 (PDB ID: 1XR8) as a template, with a sequence identity of $98.19 \%$. The protein sequence of HLA-B*15:02/HLAB*15:01 (Fig. 1) was aligned using ClustalW. Further, the homology model generated by MODELLER was selected based on the lowest DOPE score. The DOPE score for HLA-B*15:02 was -28405.27 .

\section{Evaluation of model quality}

The model with the lowest DOPE score was selected and subjected for quality evaluation. The quality of the predicted model was evaluated using RAMPAGE. Fig. 2a shows Ramachandran plot generated by RAMPAGE with most of the residues in the favorable zones i.e. the allowed regions for HLA-B*15:02 model. The Ramachandran plot analysis for HLA-B*15:02 shows that the residues in the most favored region are $98.2 \%$, and the residues in allowed region are $1.5 \%$.

Further model validation was carried out using PROCHECK based G-factors that indicates the quality of covalent and bond-angle distance. The Gfactors, indicating the quality of covalent and bond angle distance, were 0.01 for dihedrals, 0.41 for covalent, and overall 0.18 for HLA-B*15:02. The main chain bond length was $100 \%$ while the main chain bond angle was $98 \%$ within the limits for HLA-B*15:02. The overall main-chain and sidechain parameters as evaluated by PROCHECK were favorable for HLA-B*15:02. The modeled structure of HLA-B*15:02 is shown in (Fig. 2b). 
Thus, the modeled structure was reliably employed for further studies.

HLA-B*15:02 belong to the same MHC class I that have domains such as $\alpha_{1}$-domain, $\alpha_{2}$-domain, $\beta$ pleated sheets and $\alpha_{3}$-domain. The antigen or the peptide binding groove is located at the interface of $\alpha_{1}$ and $\alpha_{2}$ domain, beneath the $\beta$-pleated sheet. In HLA-B*15:01 the guanidine group of ARG62 forms salt bridge with the carboxylate of GLU63. While GLU63 mutation of to ASN63 in HLA$\mathrm{B}^{*}$ 15:02 does not allow salt bridge formation. As a result, the HLA-B*15:02 antigen binding sites remain unaffected, and the mutation of this specific amino acid might be crucial for the specificity of the peptide binding or the antigen recognition at the B-pocket (Fig. 3a).

Table 1. Demographic characteristic among patients

\begin{tabular}{|c|c|c|c|c|}
\hline Characteristics & $H L A-B * 15: 02$-Positive & $H L A-B * 15: 02$-Negative & Total & $p$ \\
\hline Sex - number $(\%)$ & & & & 0.092 \\
\hline Male & $5(15.2 \%)$ & $28(84.8 \%)$ & $33(38.37 \%)$ & \\
\hline Female & $16(30.2 \%)$ & $37(69.8 \%)$ & $53(61.63 \%)$ & \\
\hline Total & $21(24.4 \%)$ & $65(75.6 \%)$ & $86(100 \%)$ & \\
\hline Race - number (\%) & & & & 0.181 \\
\hline Malay & $12(33.3 \%)$ & $24(66.7 \%)$ & $36(41.86 \%)$ & \\
\hline Chinese & $5(19.2 \%)$ & $21(80.8 \%)$ & $26(32.23 \%)$ & \\
\hline Indian & $1(6.7 \%)$ & $14(93.3 \%)$ & $15(17.44 \%)$ & \\
\hline Others & $3(33.3 \%)$ & $6(66.7 \%)$ & $9(10.47 \%)$ & \\
\hline Total & $21(24.4 \%)$ & $65(75.6 \%)$ & $86(100 \%)$ & \\
\hline Indication for & & & & 0.180 \\
\hline Carbamazepine - number (\%) & & & & \\
\hline Epilepsy & $16(25.8 \%)$ & $46(74.2 \%)$ & $62(72.09 \%)$ & \\
\hline Neuropathic pain & $2(11.8 \%)$ & $15(88.2 \%)$ & $17(19.77 \%)$ & \\
\hline Trigeminal neuralgia & $1(100 \%)$ & 0 & $1(1.16 \%)$ & \\
\hline Other condition & $2(33.3 \%)$ & $4(66.7 \%)$ & $6(6.98 \%)$ & \\
\hline Total & $21(24.4 \%)$ & $65(75.6 \%)$ & $86(100 \%)$ & \\
\hline
\end{tabular}

Table 2. Occurrence of ADR respect to their genotypes in patients with retrospective recruitment

\begin{tabular}{|c|c|c|c|c|c|c|}
\hline \multirow[t]{2}{*}{ Patients } & \multicolumn{2}{|c|}{$H L A-B * 15: 02$ Negative } & \multicolumn{2}{|c|}{$H L A-B * 15: 02$ Positive } & \multirow[t]{2}{*}{ Total } & \multirow[t]{2}{*}{$\boldsymbol{P}^{\#}$} \\
\hline & Prospective & Retrospective & Prospective & Retrospective & & \\
\hline No ADR & 32 & 22 & 8 & 1 & 63 & $0.0002^{\wedge}$ \\
\hline Rash & 0 & 9 & 0 & 4 & 13 & \\
\hline SJS & 0 & 0 & 0 & 9 & 9 & \\
\hline TEN & 0 & 0 & 0 & 1 & 1 & \\
\hline Total & 32 & 31 & 8 & 15 & 86 & \\
\hline
\end{tabular}


HLA $A-B * 1502$

HLA-B*1501

HLA-B* 1502

HLA $-B \star 1501$

HLA-B* 1502

HLA-B* 1501

HLA-B $* 1502$

HLA-B* 1501

HLA $-B * 1502$

HLA-B*1501

GSHSMRYFYTAMSRPGRGEPRFIAVGYVDDTQFVREDSDAASPRMAPRAPWIEQEGPEYW 60

GSHSMRYFYTAMSRPGRGEPRFIAVGYVDDTQFVRFDSDAASPRMAPRAPWIEQEGPEYW 60

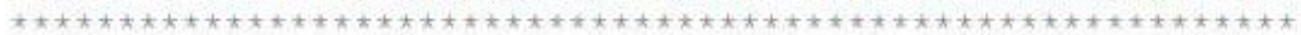

DENTTISKTNTQTYRESLRNLRGYYNQSEAGSFITTRMYGCDVGPDGRLLRGMDSAYDG 120 DEETQISKTNTQTYRESLRNLRGYYNQSEAGSH 4 HRMYGCDVGPDGRLLRGEDSAYDG 120

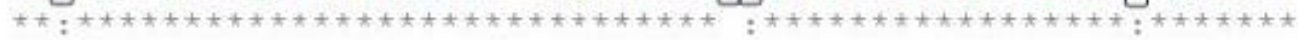

KDYIALNEDLSSWTAADTAAQITQRKWEAAREAEOIRAYLEGLCVEWLRRYLENGKETLQ 180 KDYIALNEDLSSWTAADTAAQITQRKWEAAREAEQWRAYLEGLCVEWLRRYLENGKETLQ 180

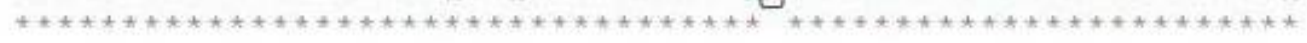

RADPPKTHVTHHPISDHEATLRCWALGFYPAEITLTWQRDGEDQTQDTELVETRPAGDRT 240 RADPPKTHVTHHPISDHEATLRCWALGFYPAEITLTWQRDGEDQTQDTELVETRPAGDRT 240

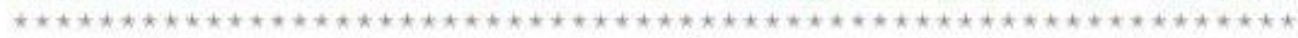

\section{FQKWAAVVVPSGEEQRYTCHVQHEGLPKPLTLRWEP 276}

EQKWAAVVVPSGEEQRYTCHVQHEGLPKPLTLRWEP 276

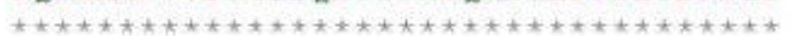

Figure 1. Protein sequence alignment of HLA-B*15:02/HLA-B*15:01in ClustalW. Protein sequence alignment of HLA$\mathrm{B}^{* 15: 02}$ and HLA-B*15:01. Variable residues are shown in box.

$\mathbf{a}$

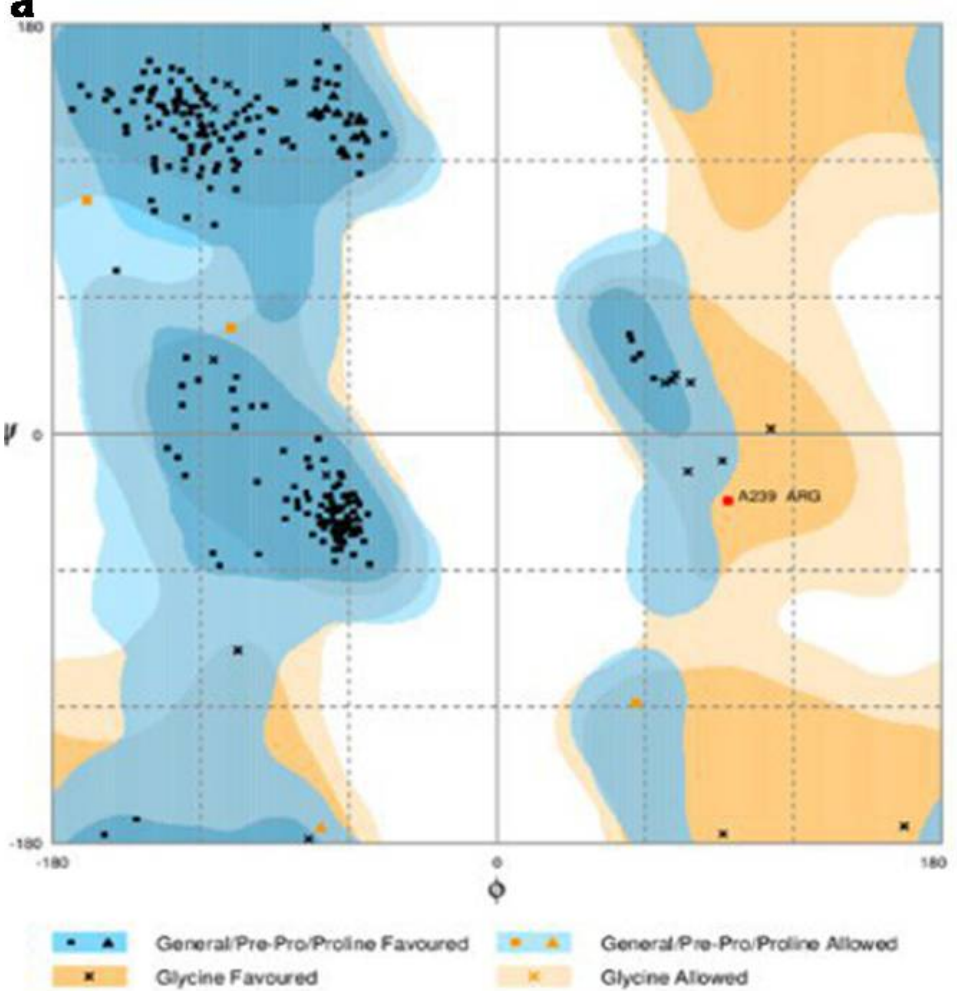

b

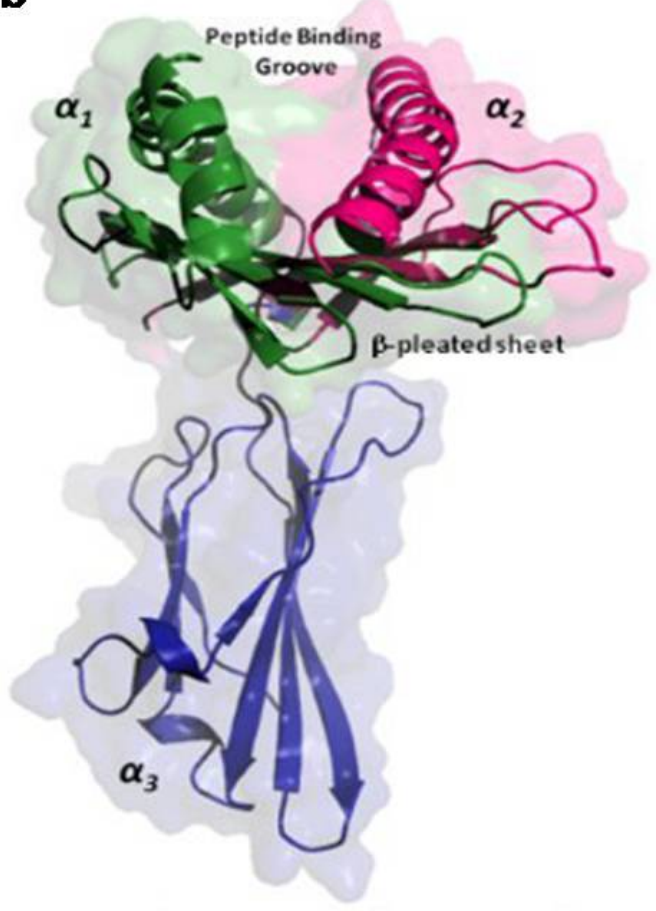

Figure 2. Ramachandran plot and homology model of HLA-B*15:02. (a) Ramachandran plot of HLA-B*15:02 model. The blue, light blue, brown and pale brown shaded regions correspond to general favored regions, general glycine favored region, glycine allowed region and disallowed regions. (b) Homology modeled structure of HLA-B*15:02 in cartoon with the $\alpha_{1}$-domain in green, $\alpha_{2}$-domain in magenta and $\alpha_{3-}$ domain in blue color. 

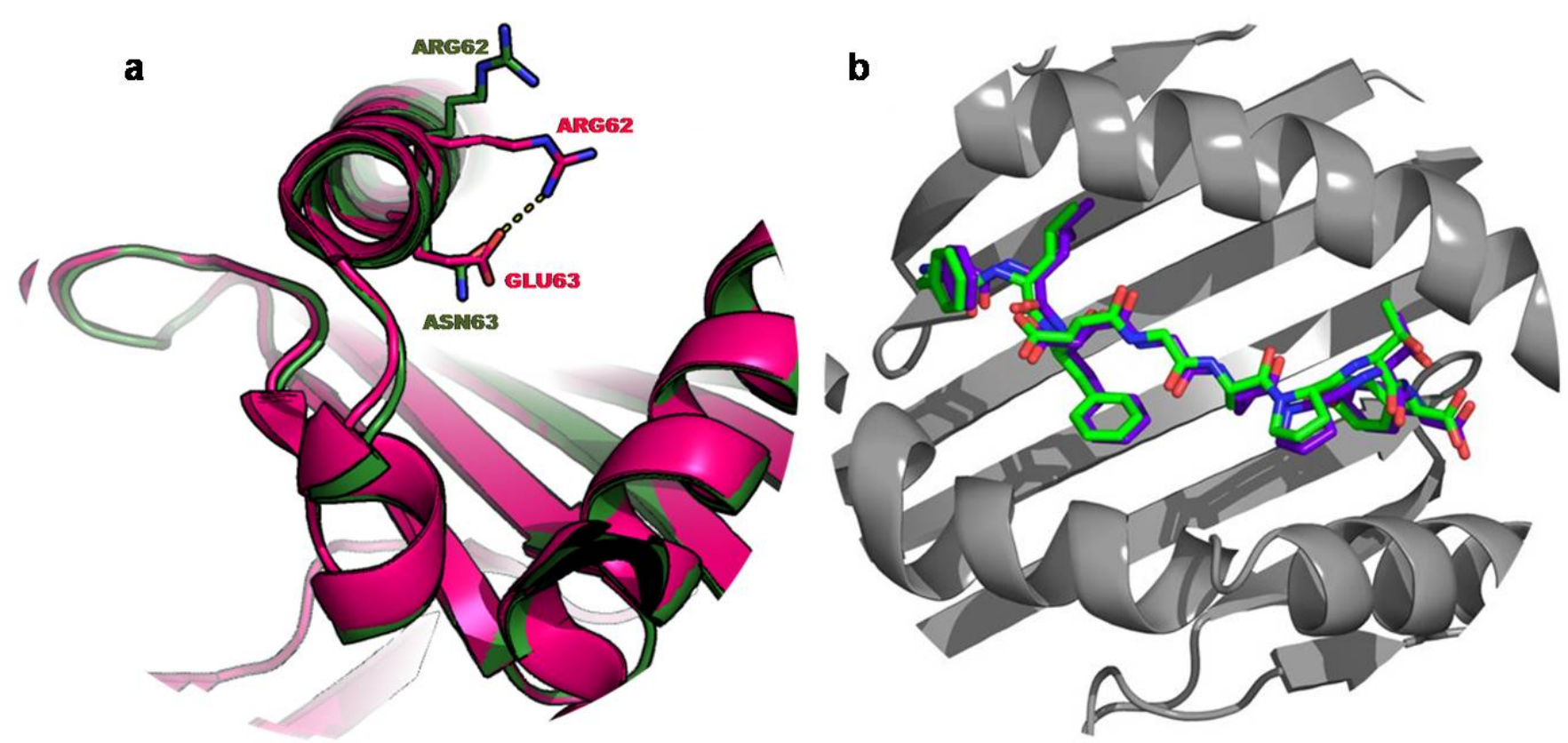

Figure 3. (a) Comparison of key residues in the B-pocket of HLA-B*15:01 and HLA-B*15:02.B-pocket of HLA-B*15:01, HLA-B*15:02 shown in magenta and green colors, respectively. Residues ARG62, GLU63and ASN63 are represented as sticks. (b) Comparison of binding modes for HLA-B*15:02/FLFDGSPTY complex from FlexPepDock and PatchDock/FireDock. Binding modes of peptide FLFDGSPTY green stick (FlexPepDock) and violet stick (PatchDock/FireDock) in HLA-B*15:02 (gray cartoon). Peptide is represented as stick and HLA- B*15:02 as cartoon.

\section{Modeling HLA-B*15:02 peptide-loaded complex}

The HLA-B*15:02/FLFDGSPTY peptide complex was modeled based on the peptide (LEKARGSTY) complexed with the crystal structure of HLA$\mathrm{B}^{*} 15: 01$ as a reference. The peptide loaded complex was optimized using FlexPepDock, a protein-peptide docking server, which generated top 10 protein-peptide complexes for each input complex. Among them, the best complex was selected entirely based on the best full-atom energy and subsequently compared to their corresponding reference peptide binding mode. The top full-atom total energy score of the best complex of HLAB*15:02/FLFDGSPTY was -584.611. The resulting top full-atom total energy scored complex was considered for further studies. To cross validate the FlexPepDock results, PatchDock and FireDock studies were performed, and the ten best docked solutions were selected from refinement and rescoring analysis. For the best docking model of HLA-B*15:02/FLFDGSPTY binding energy was 108.04. FlexPepDock and PatchDock/FireDock docked binding modes were in agreement to each other for the complex (Fig. 3b).
All the 26 AEDs structures (Fig. 6) were docked using GOLD that predicts the binding of all the AEDs with the antigen binding groove of the peptide-loaded complex. The results of the docking studies were analyzed for the binding ability of AEDs that plausibly may induce ADR. Given the fact that both HLA-B*15:02 involve in CBZ induced ADR, molecular level interaction study reveals their feasibility to induce hypersensitivity upon CBZ binding, albeit they belong to different HLA subtypes. The docked ChemScore function for CBZ on B-pocket for HLA-B*15:02/FLFDGSPTY complex was 27.77. The ChemScore and CBZ binding mode of HLA-B*15:02/FLFDGSPTY complexes was in agreement upon analysis and, therefore, this complex was considered for further studies.

\section{The CBZ binding mode in $H L A$ -} $B * 15: 02 / F L F D G S P T Y$ complex

The docked binding mode of CBZ within the Bpocket/antigen binding groove of HLAB*15:02/FLFDGSPTY complex forms a stable interaction. The ketone group of 5-carboxamide of CBZ forms a hydrogen bond with the guanidine 
group of ARG62, the tricyclic ring of CBZ forms a hydrophobic interaction with the side chain ring of TYR59, indole ring of TRP167 and the aliphatic chain of LEU163. Interestingly, the tricyclic ring of CBZ forms T-shaped $\pi-\pi$ stacking with PHE1 of FLFDGSPTY-nonameric peptide. Geometrically, the tri-cyclic ring of CBZ fits well in the B-pocket of HLA-B*15:02 (Fig. 4a).

\section{Docking studies of 26 AEDs with HLA- $B *$ 15:02/FLFDGSPTY complex}

In the second phase of the study, all the 26 AEDs were docked to HLA-B*15:02/FLFDGSPTY complex to screen the AEDs that could bind in a similar fashion alike CBZ and to be possible putative candidate that that may induce SCAR among the carriers of HLA-B*15:02. Following the docking protocol, analyzes were done based on ChemScore and visually inspection in PyMOL. Among the 26 AED's eight were selected based on their interaction pattern. Aacetazolamide, ethosuxiamide, lamotrigine, oxcarbazepine, phenobarbital, phenytoin, primidone and sodiumvalproate were selected (Fig. 4b).

Out of the eight AEDs, ethosuxiamide, oxcarbazepine, phenobarbital, phenytoin, primidone, and sodium-valproate shows similar binding mode to that of CBZ, as their structural scaffolds are similar. While acetazolamideand lamotrigine despite having a different chemical scaffold to that of CBZ yet shows a stable interaction at binding B pocket alike CBZ.

In acetazolamide, the sulfamoyl group oxygen forms hydrogen bond with the guanidine $\mathrm{NH}$ of ARG62. While the NH forms hydrogen bond with carboxyl oxygen GLU58 and the acetamide group forms a hydrogen bond with the guanidine $\mathrm{NH}$ of ARG170. While LEU163 forms hydrophobic interaction with the methyl group of acetamide as shown in (Fig. 5a).

In the case of ethosuxiamide, the pyrrolidine oxygens form a hydrogen bond with the guanidine $\mathrm{NH}$ of ARG62 and with the guanidine $\mathrm{NH}$ of ARG170 respectively. The ethyl group of ethosuxiamide forms hydrophobic interaction with LEU163 and PHE1 phenyl group of the FLFDGSPTY peptide as shown in (Fig. 5b).

In the case of lamotrigine, dichlorophenylring forms hydrophobic interaction with PHE1 of FLFDGSPTY-nonameric peptide, while the amine attached at the ortho position of triazine forms hydrogen bond with carboxyl oxygen of Glu58. The triazine ring forms hydrophobic interaction with TYR59, indole ring of TRP167 and aliphatic chain of LEU163 (Fig.5c).Oxcarbazepine binding mode shows that the hydroxy group attached to the azepine ring forms hydrogen bond with the guanidine group of ARG170, while the carboxamide oxygen forms hydrogen bond with the guanidine NH of ARG62. Additionally the tricyclic ring of oxcarbazepine forms hydrophobic interaction with the side chain ring of TYR59, indole ring of TRP167 and aliphatic chain of LEU163. Interestingly the tricyclic ring of oxcarbazepine forms T-shaped $\pi-\pi$ stacking with PHE1 of FLFDGSPTY-nonameric peptide. (Fig.5d). While in the case of phenobarbital, the guanidine $\mathrm{NH}$ of ARG62 and ARG170 forms hydrogen bond with oxygen attached to the phenylpyrimidine trione, and the TYR59, TRP167, LEU163 and PHE1 of FLFDGSPTY forms hydrophobic interaction with phenylpyrimidine trione ring (Fig.5e). Whereas, in the case of phenytoin, the guanidine $\mathrm{NH}$ of ARG62 and ARG170 forms hydrogen bond with oxygen attached to imidazolidine, and the TYR59, TRP167, LEU163 and FLFDGSPTY PHE1 of peptide forms hydrophobic interaction with diphenylimidazolidine as shown in (Fig.5f). In the case of primidone the diazinane-4, 6-dione oxygen forms hydrogen bond with the guanidine NH of ARG62, while the phenyl ring of benzodiazepine forms $\pi$ - $\pi$ stacking with the indole group of TRP167 and hydrophobic interaction with PHE1 of FLFDGSPTY peptide, while the ethyl group forms hydrophobic interaction with LEU163 side chain as shown in (Fig.5g). In case of sodium-valproate the pentanoate oxygen forms hydrogen bond with the guanidine $\mathrm{NH}$ of ARG170, where the propyl group forms hydrophobic interaction with TRP167 and the phenyl group of PHE1 of FLFDGSPTY peptide (Fig.5h).

The current computational study shows the identification of AEDs specific molecular interaction patterns to differentiate between inducer and non-inducer of SJS using CBZ interaction pattern as a reference point. 


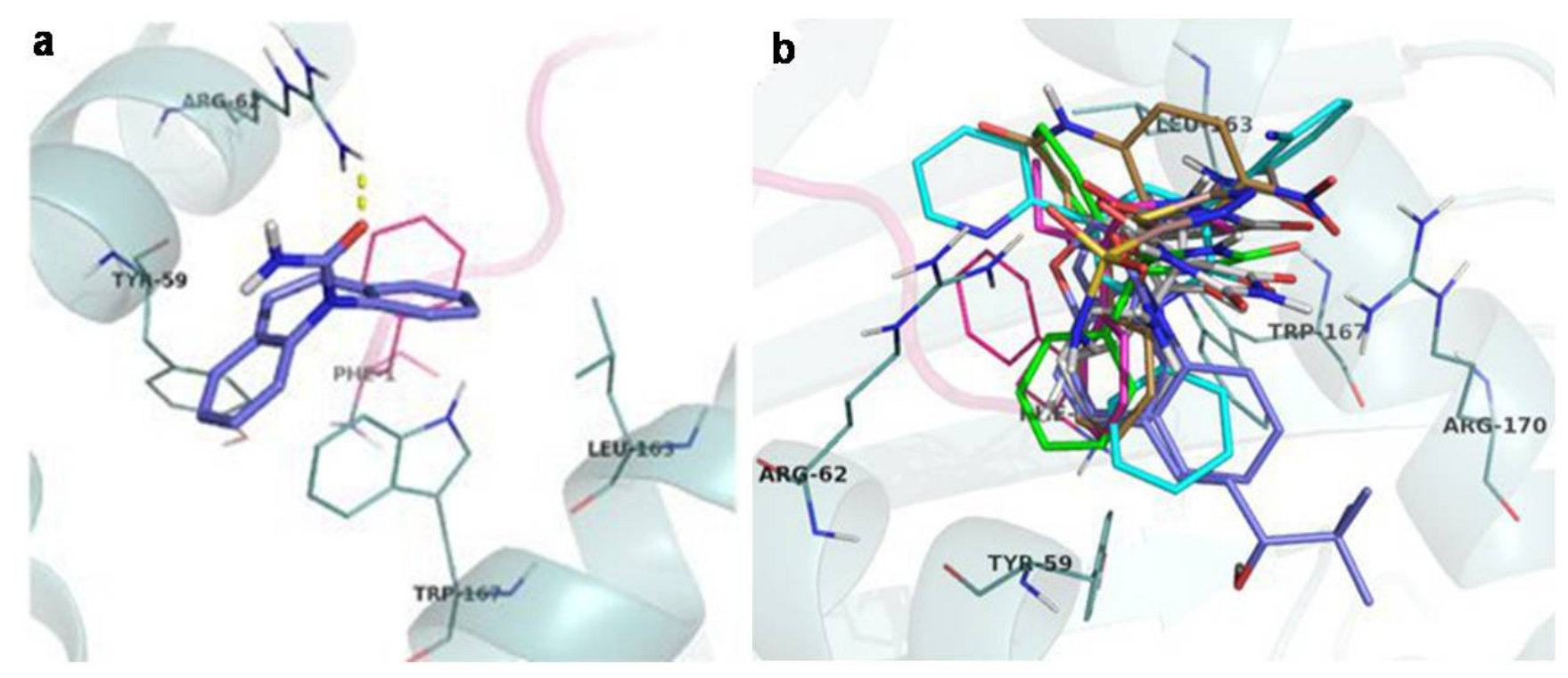

Figure 4. (a) Interaction of CBZ in the peptide loaded HLA-B*15:02 complex. Bound state of CBZ (purple blue color) in the HLA-B*15:02/FLFDGSPTY peptide complex. The yellow dashed line represents the hydrogen bond between ARG62 of HLA-B*15:02and the ketone group of CBZ and the other side chains engaged in hydrophobic interactions are shown in cyan color lines. The magenta colored line represents the PHE1 of FLFDGSPTY peptide that forms hydrophobic interaction with the cyclic ring of CBZ. (b) Predicted binding modes of 26 AEDs (stick) in the HLA-B*15:02/FLFDGSPTY complex.

\section{DISCUSSION}

A study report in 2010 indicated that carbamazepine, allopurinol and cotrimoxazole were the three main drugs which cause SJS/TEN in Malaysia. These accounted for $24.0 \%, 18.8 \%$ and $12.5 \%$, of all ADR cases reported from 2001 to 2008 in Malaysia [31]. Therefore, there is a continuous interest among different groups of researchers in Malaysia to determine causative agents associated with these incidences. This is in line with the strategies to improve the quality of patients care and in realizing personalized medicine in Malaysia. Subsequent to data published by Chung et al [11] and Man et al [12], the association of $H L A-B^{*} 1502$ genetic marker with CBZ induced SJS/TEN was reported by Chang et al [32]. Whereby 21 subjects with CBZ-induced TEN/SJS were studied. However, $H L A-B * 15: 02$ was present in $75.0 \%(12 / 16)$ of the Malay patients who developed SJS/TEN resulting in an odds ratio 16.15, 95\% confidence interval 4.57-62.4; corrected $\mathrm{P}$-value $=7.87 \times 10\left(^{-6}\right)$; while we reported a higher odd ratio of 609.0 (95\% CI: $23-15873 ; p=0.001)$. In our study, 14 out of 15 patients who were positive for $H L A-B * 15: 02$ developed SJS/TEN, leaving one patient being tolerant to $\mathrm{CBZ}$ despite being positive of $H L A-B^{*} 15: 02$. No patients who were negative for $H L A-B * 15: 02$ developed SJS/TEN. The diverged result could be explained by the use of different detection method. We therefore hypothesised that use of sensitive and specific method is very important to avoid false negative. Similarly high frequency of $H L A-$ $B *$ 15:02 allele in CBZ induced SJS-TEN (94.1\%) was reported in Thailand [33,34]. In India, eight individuals who fulfilled the diagnostic criteria of SJS induced by carbamazepine were identified and $H L A-B$ molecular typing was performed. Out of eight patients, six patients were found to have the $H L A-B * 15: 02$ allele [35].A meta-analysis conducted by Tangamornsuksan et al [13] revealed a high risk of developing SJS and TEN among carbamazepine users with $H L A-B * 15: 02$. For HanChinese, Thais, Koreans, and Malaysians, the risks of SJS and TEN among carbamazepine users carrying the $H L A-B * 15: 02$ allele are approximately 115-fold, 60-fold, 25-fold, and 220-fold higher, respectively. 


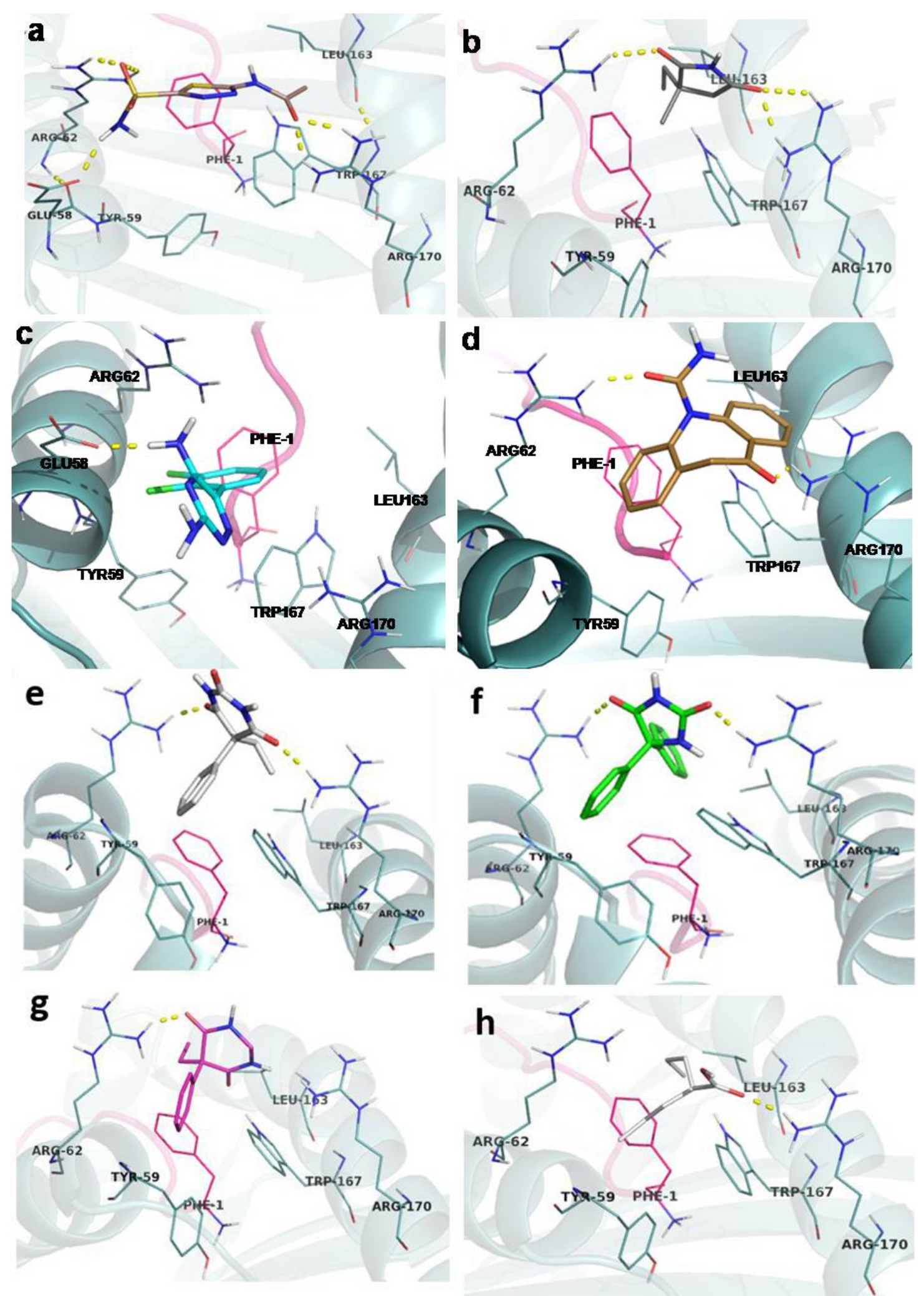

Figure 5. Graphical illustration of predicted binding mode of AEDs in the HLA-B*15:02/FLFDGSPTY complex. a. acetazolamide (pale brown color) b. ethosuxiamide (gray color), c.lamotrigine (cyan color), d. Oxcarbazepine (brown color), e. phenobarbital (pale gray color), f. phenytoin (green color), g. primidone (magenta color), h. sodium-valproate in silver color. Key residues are only shown and the hydrogen bond interactions are represented by yellow dashed lines. Compounds are shown as sticks and key amino acids as lines. 


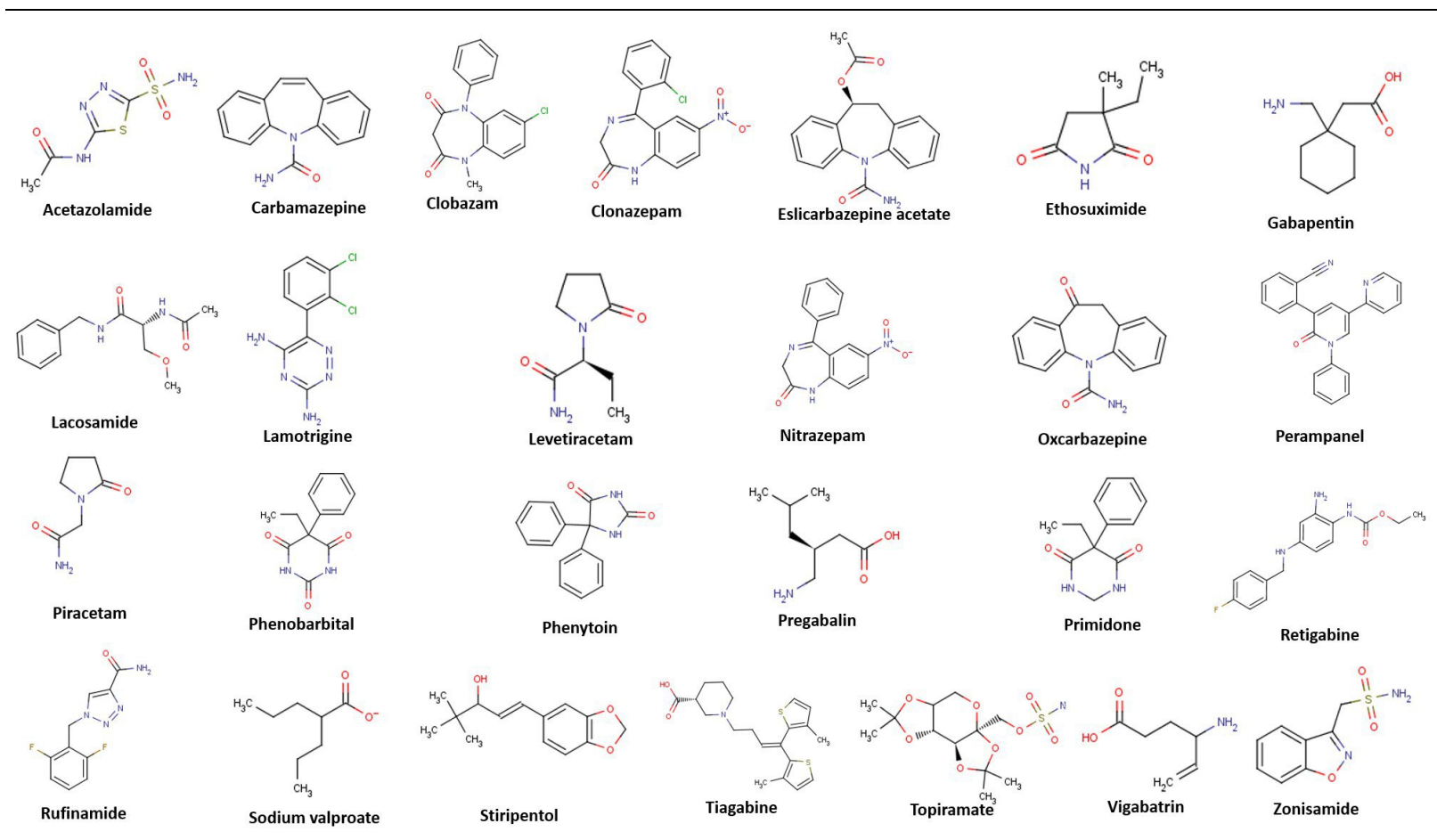

Figure 6. Chemical structures of 26 AEDs

With respect to the prevalence of $H L A-B * 15: 02$, Chang et al [32] reported the presence of $H L A$ $B^{*} 15: 02$ in $15.7 \%(47 / 300)$ of normal controls. We reported the presence of the allele in $17.5 \%$ of patients who requires CBZ therapy. A study that recruited 56 Caucasian patients who developed serious hypersensitivity reaction which include SJS/TEN found that none of the patients carry $H L A$ $B * 15: 02$ [36]. Another study identified twelve cases of CBZ-induced SJS/TEN (retrieved from RegisSCAR). And only 4 of the patients were positive for $H L A-B^{*} 15: 02$ allele. However, those four positive $H L A-B^{*}$ 15:02 allele were from Asian ancestry [14]. Therefore, $H L A-B * 15: 02$ is not a marker of SJS/TEN among the Caucasian and other alleles but could be responsible for the occurrence of $\mathrm{CBZ}$ related hypersensitive reactions in these populations. The search for genetic markers therefore continues. The $H L A-A * 31: 01$ allele which has a prevalence of 2 to $5 \%$ in the Northern European populations was later found to be significantly associated with the $\mathrm{CBZ}$ related hypersensitivity syndrome $\left(\mathrm{P}=3.5 \times 10^{-8}\right)$. Genotyping confirmed the variant as a risk factor for the hypersensitivity syndrome (odds ratio, $12.41 ; 95 \%$ confidence interval [CI], 1.27 to 121.03), maculopapular exanthema (odds ratio,
8.33; 95\% CI, 3.59 to 19.36), and SJS-TEN (odds ratio, 25.93; $95 \% \mathrm{CI}, 4.93$ to 116.18 ) [36]. However, $H L A-B^{*} 15: 02$ is rare in the Japanese population and is not found in patients with SJS induced by CBZ[15]. Further research by Ikeda et al. suggested that $H L A-B * 59: 01$ is one of the candidate markers for CBZ-induced SJS in Japanese [16].

Interestingly, there were 9 patients who developed mild rashes among the patients, but they were negative for $H L A-B * 15: 02$. Similar to a study conducted earlier by another research group in Malaysia, Then et al. found 6 out of 27 patients who developed skin rashes after CBZ administration were not positive for the $H L A-B * 15: 02$ allele [37]. Therefore, further research is required to determine alleles that caused skin rashes other than HLA$B * 15: 02$.

Our results indicate that the $H L A-B * 15: 02$ allele is strongly associated with increased risk of developing SJS and TEN in patients using carbamazepine and further justified the clinical practice of pre-genotyping in Malaysian. This severe adverse event could be prevented if genetic information were known a priori. Physicians and policymakers should consider our findings to support the implementation of genetic testing before 
initiation of carbamazepine therapy. In addition to the clinical importance, the availability of simple and rapid genotyping kit is necessary to implement pharmacogenotyping of $H L A B * 15: 02$ in clinics. Currently, the method adopted in this study allows identification of patients at risk within 4 hours and using the method optimized and validated in our study. We were able to detect patients with $H L A$ $B * 15: 02$ correctly. The genotype was reconfirmed with direct DNA sequencing technique by a third party. The prospective part of the study further shows that pharmacogenotyping is important as $H L A-B^{*} 15: 02$ is relatively common in the Malaysian population. Clinicians are recommended to refer to the clinical pharmacogenetics implementation consortium guidelines for the use of carbamazepine based on HLA-B genotype [38].

In the present computational study, 26 AEDs have been virtually screened to identify AEDs that may cause ADR similar to that of CBZ, specific to $H L A-B * 15: 02$. The computational interaction study clearly discriminates between the inducers from non-inducers of ADR among $H L A-B^{*} 15: 02$ carriers. Our study also reveals that the preloaded endogenous peptide specificity is crucial for the AEDs induction and stabilization in order to cause ADR. This study also suggests that apart from hydrogen bonding, hydrophobic interaction is also crucial for stabilization of the complex to induce ADR. This hypothetical molecular interaction study attempts to reveal, how some AEDs act antigenic in some individuals to induce drug mediated hypersensitivity. The current computational modeling study also demonstrates that the presence of specific endogenous peptide in theHLA-B*15:02 is essential to stabilize the complex, which in turn would be bound by the AEDs to induce hypersensitivity. Although the stability of the complex is important, the conformational orientation of the peptide also plays a crucial role in determining the drug interaction with the peptide mediated complex. Our modeling result also shows that the residue ARG62 at the B pocket is the key residue for interaction with the AEDs.

Our computational studies show that acetazolamide, ethosuxiamide, lamotrigine, oxcarbazepine, phenobarbital, phenytoin, primidone and sodium-valproate may cause ADR similar to that of CBZ in Southeast Asians carrying HLA$B * 15: 02$ allele. AEDs like clobazam, clonazepam, Eslicarbazepine acetate, gabapentin, lacosamide, levetiracetam, nitrazepam, perampanel, piracetam, pregabalin, retigabine, rufinamide, stiripentol, tiagabine, topiramate, vigabatrin, and zonisamide did not show stable binding mode. The key chemical moiety attached to the core scaffold of the above AEDs results in unfavorable hydrogen bonding and hydrophobic interaction with the peptide loaded complex that affects their binding affinity. Henceforth, the study theoretically shows that these AEDs as non-binders which could be proposed as safe AEDs among individuals who develop drug induced ADR after clinical confirmation. However, additional clinical studies may be required to confirm the AEDs inducing ADR among $H L A-B *$ 15:02 allele carriers.

\section{CONCLUSION}

Screening patients for the $H L A-B * 15: 02$ allele before the initiation of carbamazepine treatment and withholding carbamazepine from $H L A-B * 15: 02-$ positive patients is important clinically to reduce the incidence of carbamazepine-induced SJS/TEN among the Malaysians. Pharmacogenotyping based practice of $H L A-B * 15: 02$ should be adopted by clinician as part of routine patient care. A new perspective for screening HLA-associated drug hypersensitivity using molecular docking technique was proposed in the present study. This approach will help to select safe AEDs for individuals with $H L A-B * 15: 02$ allele. This approach is particularly important if HLA polymorphism is widely present among the population.

\section{ACKNOWLEDGEMENTS}

The project is funded by Ministry of Education Malaysia (600/RMI/TRGS/5/3(1/2014)-3). The authors would like to extend gratitude to the participation of the patients in this study as well as the involvement of nurses, pharmacists and doctors from Hospital Kuala Lumpur in recruitment of patients. We also acknowledge the Director General of Ministry of Health in support of this study.

\section{CONFLICT OF INTEREST}

None of the authors declared any conflicts of interest. 


\section{REFERENCES}

1. Attia SM. Deleterious effects of reactive metabolites. Oxidative medicine and cellular longevity 3(4), 238-253 (2010) doi: 10.4161/oxim.3.4.13246.

2. Yuksel A, Cengiz M, Seven M, Ulutin T. Erythrocyte glutathione, glutathione peroxidase, superoxide dismutase and serum lipid peroxidation in epileptic children with valproate and carbamazepine monotherapy. Journal of basic and clinical physiology and pharmacology 11(1), 73-81 (2000).

3. Sobaniec W, Solowiej E, Kulak W, Bockowski L, Smigielska-Kuzia J, Artemowicz B. Evaluation of the influence of antiepileptic therapy on antioxidant enzyme activity and lipid peroxidation in erythrocytes of children with epilepsy. Journal of child neurology 21(7), 558-562 (2006).

4. Aycicek A, Iscan A. The effects of carbamazepine, valproic acid and phenobarbital on the oxidative and antioxidative balance in epileptic children. European neurology 57(2), 65-69 (2007).

5. Naisbitt DJ, Britschgi $M$, Wong $G$ et al. Hypersensitivity reactions to carbamazepine: characterization of the specificity, phenotype, and cytokine profile of drug-specific $\mathrm{T}$ cell clones. Molecular pharmacology 63(3), 732-741 (2003).

6. Padovan E, Bauer T, Tongio MM, Kalbacher H, Weltzien HU. Penicilloyl peptides are recognized as $\mathrm{T}$ cell antigenic determinants in penicillin allergy. European journal of immunology 27(6), 1303-1307 (1997).

7. Pichler WJ. Delayed drug hypersensitivity reactions. Annals of internal medicine 139(8), 683-693 (2003).

8. Fruci D, Rovero P, Butler RH, Sorrentino R, Tosi R, Tanigaki N. HLA class I binding of synthetic nonamer peptides carrying major anchor residue motifs of HLA-B27 (B*2705)-binding peptides. Immunogenetics 38(1), 41-46 (1993).

9. Illing PT, Vivian JP, Dudek NL et al. Immune selfreactivity triggered by drug-modified HLA-peptide repertoire. Nature 486(7404), 554-558 (2012). doi: 10.1038/nature11147.

10. Pirmohamed M, Lin K, Chadwick D, Park BK. TNFalpha promoter region gene polymorphisms in carbamazepine-hypersensitive patients. Neurology 56(7), 890-896 (2001).

11. Chung WH, Hung SI, Hong HS et al. Medical genetics: a marker for Stevens-Johnson syndrome. Nature 428(6982), 486 (2004).

12. Man CB, Kwan P, Baum L et al. Association between HLA-B*1502 allele and antiepileptic druginduced cutaneous reactions in Han Chinese. Epilepsia 48(5), 1015-1018 (2007).

13. Tangamornsuksan W, Chaiyakunapruk N, Somkrua R, Lohitnavy M, Tassaneeyakul W. Relationship between the HLA-B*1502 allele and carbamazepine-induced Stevens-Johnson syndrome and toxic epidermal necrolysis: a systematic review and meta-analysis. JAMA dermatology 149(9), 1025-1032 (2013). doi: 10.1001/jamadermatol.2013.4114.

14. Lonjou C, Thomas L, Borot $\mathrm{N}$ et al. A marker for Stevens-Johnson syndrome ...: ethnicity matters. The pharmacogenomics journal 6(4), 265-268 (2006).

15. Kashiwagi M, Aihara M, Takahashi Y et al. Human leukocyte antigen genotypes in carbamazepineinduced severe cutaneous adverse drug response in Japanese patients. The Journal of dermatology 35(10), 683-685 (2008). doi: 10.1111/j.13468138.2008.00548.x

16. Ikeda H, Takahashi Y, Yamazaki E et al. HLA class I markers in Japanese patients with carbamazepineinduced cutaneous adverse reactions. Epilepsia 51(2), 297-300 (2010). doi: 10.1111/j.15281167.2009.02269.x. Epub 2009 Aug 19.

17. CPIC Dosing Guideline for carbamazepine and HLA-B. 2014(Jan), (2001).

18. Reorganizing the protein space at the Universal Protein Resource (UniProt). Nucleic acids research 40(Database issue), D71-75 (2012). doi: 10.1093/nar/gkr981. Epub 2011 Nov 18.

19. Roder G, Blicher T, Justesen $\mathrm{S}$ et al. Crystal structures of two peptide-HLA-B*1501 complexes; structural characterization of the HLA-B62 supertype. Acta crystallographica. Section D, Biological crystallography 62(Pt 11), 1300-1310 (2006).

20. Sali A, Blundell TL. Comparative protein modelling by satisfaction of spatial restraints. Journal of molecular biology 234(3), 779-815 (1993).

21. Morris AL, Macarthur MW, Hutchinson EG, Thornton JM. Stereochemical quality of protein structure coordinates. Proteins 12(4), 345-364 (1992).

22. Wei CY, Chung WH, Huang HW, Chen YT, Hung SI. Direct interaction between HLA-B and carbamazepine activates $\mathrm{T}$ cells in patients with Stevens-Johnson syndrome. The Journal of allergy and clinical immunology 129(6), 1562-1569.e1565 (2012). doi: 10.1016/j.jaci.2011.12.990. Epub 2012 Feb 8.

23. London N, Raveh B, Cohen E, Fathi G, SchuelerFurman O. Rosetta FlexPepDock web server--high resolution modeling of peptide-protein interactions. Nucleic acids research 39(Web Server issue), W249253 (2011). doi: 10.1093/nar/gkr431. Epub 2011 May 27.

24. Raveh B, London N, Schueler-Furman O. Subangstrom modeling of complexes between flexible peptides and globular proteins. Proteins 78(9), 20292040 (2010). doi: 10.1002/prot.22716.

25. Schneidman-Duhovny D, Inbar Y, Nussinov R, Wolfson HJ. PatchDock and SymmDock: servers for 
rigid and symmetric docking. Nucleic acids research 33(Web Server issue), W363-367 (2005).

26. Andrusier N, Nussinov R, Wolfson HJ. FireDock: fast interaction refinement in molecular docking. Proteins 69(1), 139-159 (2007).

27. Zhang C, Vasmatzis G, Cornette JL, Delisi C. Determination of atomic desolvation energies from the structures of crystallized proteins. Journal of molecular biology 267(3), 707-726 (1997).

28. List of anti-epileptic drugs. 2013(22 Sep), (2013).

29. Verdonk ML, Cole JC, Hartshorn MJ, Murray CW, Taylor RD. Improved protein-ligand docking using GOLD. Proteins 52(4), 609-623 (2003).

30. PyMOL Molecular Graphics System, L., NY, USA, . PyMOL 0.99; Schrçdinger: New York, US,. PyMOL 0.992013 ( 2010).

31. Ding WY, Lee CK, Choon SE. Cutaneous adverse drug reactions seen in a tertiary hospital in Johor, Malaysia. International journal of dermatology 49(7), 834-841 (2010). doi: 10.1111/j.13654632.2010.04481.x.

32. Chang CC, Too CL, Murad S, Hussein SH. Association of HLA-B*1502 allele with carbamazepine-induced toxic epidermal necrolysis and Stevens-Johnson syndrome in the multi-ethnic Malaysian population. International journal of dermatology 50(2), 221-224 (2011). doi: 10.1111/j.1365-4632.2010.04745.x.

33. Kulkantrakorn K, Tassaneeyakul W, Tiamkao S et al. HLA-B*1502 strongly predicts carbamazepineinduced Stevens-Johnson syndrome and toxic epidermal necrolysis in Thai patients with neuropathic pain. Pain practice : the official journal of World Institute of Pain 12(3), 202-208 (2012).

34. Tiamkao S, Jitpimolmard J, Sawanyawisuth K, Jitpimolmard S. Cost minimization of HLA-B*1502 screening before prescribing carbamazepine in Thailand. International journal of clinical pharmacy 35(4), 608-612 (2013).

35. Mehta TY, Prajapati LM, Mittal B et al. Association of HLA-B*1502 allele and carbamazepine-induced Stevens-Johnson syndrome among Indians. Indian journal of dermatology, venereology and leprology 75(6), 579-582 (2009). doi: 10.4103/03786323.57718 .

36. Alfirevic A, Jorgensen AL, Williamson PR, Chadwick DW, Park BK, Pirmohamed M. HLA-B locus in Caucasian patients with carbamazepine hypersensitivity. Pharmacogenomics 7(6), 813-818 (2006).

37. Mccormack M, Alfirevic A, Bourgeois S et al. HLA$A^{*} 3101$ and carbamazepine-induced hypersensitivity reactions in Europeans. The New England journal of medicine 364(12), 1134-1143 (2011). doi: 10.1056/NEJMoa1013297.

38. Then SM, Rani ZZ, Raymond AA, Ratnaningrum S, Jamal R. Frequency of the HLA-B*1502 allele contributing to carbamazepine-induced hypersensitivity reactions in a cohort of Malaysian epilepsy patients. Asian Pacific journal of allergy and immunology / launched by the Allergy and Immunology Society of Thailand 29(3), 290-293 (2011). 